\title{
Full angular profile of the coherent polarization opposition effect
}

\author{
Michael I. Mishchenko
}

NASA Goddard Institute for Space Studies, 2880 Broadway, New York, NY 10025

\section{Jean-Marc Luck}

CEA Saclay, Service de Physique Théorique, 91191 Gif-sur-Yvette Cedex, France

\author{
Theo M. Nieuwenhuizen \\ University of Amsterdam, Department of Physics and Astronomy, Valckenierstraat 65, $1018 \mathrm{XE}$ \\ Amsterdam. The Netherlands
}

We use the rigorous vector theory of weak photon localization for a semi-infinite medium composed of nonabsorbing Rayleigh scatterers to compute the full angular profile of the polarization opposition effect. The latter is caused by coherent backscattering of unpolarized incident light and accompanies the renowned backscattering intensity peak.

OCIS codes: $030.5620,260.5430,280.0280,290.1350,290.4210,290.5870$ 


\section{INTRODUCTION}

Coherent backscattering is a remarkable phenomenon caused by constructive interference of waves propagating along reciprocal multiple-scattering paths in a discrete random medium. ${ }^{1-4} \mathrm{~A}$ well-known manifestation of coherent backscattering is an intensity peak centered at exactly the backscattering direction. The magnitude of the peak relative to the incoherent background depends on the polarization states of the incident and reflected beams and can be is large as a factor of 2 when the incident beam is fully circularly polarized, the intensity of the reflected beam is measured in the same-helicity channel, and the scattering particles are spherically symmetric. $^{5}$

Another interesting and practically important manifestation of coherent backscattering can be observed when the incident beam is unpolarized. In this case the backscattering intensity peak is accompanied by a sharp asymmetric peak of negative polarization with a minimum centered at a very small phase angle (angle between the reflected beam and the backscattering direction). ${ }^{6}$ This phenomenon was called the polarization opposition effect (POE) ${ }^{6}$ and has the same physical origin as the so-called spatial anisotropy of the backscattering intensity peak in the case of fully linearly polarized incident beam. ${ }^{7}$

The calculation of the POE in Ref. 6 was based on the vector theory of coherent backscattering developed by $\mathrm{Ozrin}^{8}$ for a semi-infinite medium composed of nonabsorbing Rayleigh scatterers (particle size is much smaller than the wavelength of the incident light). Although this theory is rigorous, the final solution was given in terms of asymptotic expressions valid in the limit of very small and very large phase angles. Therefore, the full angular profile of the POE, including the exact value and the angular position of the polarization minimum, remained unknown.

In this paper, we revisit the problem of computing the full angular profile of the POE and, to this end, use the complete solution developed recently by Amic et al. ${ }^{9}$ We also compare our theoretical calculations with available laboratory data and briefly discuss remote-sensing ramifications of our results. 


\section{THEORY}

The following analysis is largely based on formulas of Ref. 9. However, since we are interested in coherent backscattering of unpolarized incident light, it is more convenient to use the standard representation of the Stokes vector rather than the modified representation adopted in Ref. 9. This change necessitates a simple linear transformation of the $4 \times 4$ matrices given by Eqs. (2.42)(2.45) of Ref. 9 as described in section 3 of Ref. 10.

Consider a semi-infinite, homogeneous random medium composed of nonabsorbing Rayleigh scatterers. We will use the standard set of Stokes parameters $I, Q, U$, and $V$ to define the polarization state of the incident and reflected beams relative to their respective meridional planes (the planes through the beams and the normal to the boundary of the medium). ${ }^{10.11}$ Assume that the medium is illuminated by a parallel beam of light incident perpendicularly to the boundary and characterized by Stokes parameters $I_{0}, Q_{0}, U_{0}$, and $V_{0}$, where $I_{0}$ is the incident energy flux per unit area perpendicular to the flux. The Stokes parameters of the beam reflected at a phase angle $\alpha$ in the meridional plane of the incident beam are given by

$$
\left[\begin{array}{l}
I \\
Q \\
U \\
V
\end{array}\right]=\frac{1}{\pi}\left[\begin{array}{cccc}
S_{11}(\alpha) & S_{12}(\alpha) & 0 & 0 \\
S_{12}(\alpha) & S_{22}(\alpha) & 0 & 0 \\
0 & 0 & S_{33}(\alpha) & 0 \\
0 & 0 & 0 & S_{44}(\alpha)
\end{array}\right]\left[\begin{array}{c}
I_{0} \\
Q_{0} \\
U_{0} \\
V_{0}
\end{array}\right]
$$

where $\mathbf{S}(\alpha)$ is the Stokes reflection matrix. ${ }^{10}$ The reflection matrix can be decomposed as ${ }^{1.5}$

$$
\mathbf{S}(\alpha)=\mathbf{S}^{1}(\alpha)+\mathbf{S}^{L}(\alpha)+\mathbf{S}^{C}(\alpha)
$$

where $\mathbf{S}^{\prime}(\alpha)$ is the first-order-scattering component, $\mathbf{S}^{\prime}(\alpha)$ is the contribution of all ladder diagrams of scattering orders 2 and higher, and $\mathbf{S}^{C}(\alpha)$ is the contribution of all cyclical diagrams of scattering orders 2 and higher. Restricting the analysis to small $\alpha$, introducing the so-called angular parameter $q=k l \alpha$, where $k$ is the wavenumber and $l$ is the photon transport mean free 
path in the scattering medium, and performing the previously mentioned matrix transformation, we derive from formulas of Ref. 9

$$
\begin{aligned}
& \mathbf{S}^{1}(\alpha) \approx \mathbf{S}^{1}(0)=\frac{3}{16}\left[\begin{array}{cccc}
1 & 0 & 0 & 0 \\
0 & 1 & 0 & 0 \\
0 & 0 & -1 & 0 \\
0 & 0 & 0 & -1
\end{array}\right] \\
& \mathbf{S}^{L}(\alpha) \approx \mathbf{S}^{L}(0) \\
& =\frac{1}{4}\left[\begin{array}{cccc}
\gamma_{11}(0)+\gamma_{12}(0) & 0 & 0 & 0 \\
0 & \gamma_{11}(0)-\gamma_{12}(0) & 0 & 0 \\
0 & 0 & \gamma_{12}(0)-\gamma_{11}(0) & 0 \\
0 & 0 & 0 & \gamma_{44}(0)
\end{array}\right]-\mathbf{S}^{1}(0), \\
& \mathbf{S}^{C}(\alpha) \approx \mathbf{S}^{C}(q)=\left[\begin{array}{cccc}
S_{11}^{C}(q) & S_{12}^{C}(q) & 0 & 0 \\
S_{12}^{C}(q) & S_{22}^{C}(q) & 0 & 0 \\
0 & 0 & S_{33}^{C}(q) & 0 \\
0 & 0 & 0 & S_{44}^{C}(q)
\end{array}\right]-\mathbf{S}^{1}(0)
\end{aligned}
$$

where

$$
\begin{aligned}
& S_{11}^{C}(q)=\frac{1}{8}\left[\gamma_{11}(q)+\gamma_{22}(q)-\gamma_{33}(q)+\gamma_{44}(q)\right], \\
& S_{22}^{C}(q)=\frac{1}{8}\left[\gamma_{11}(q)+\gamma_{22}(q)+\gamma_{33}(q)-\gamma_{44}(q)\right], \\
& S_{33}^{C}(q)=\frac{1}{8}\left[\gamma_{33}(q)+\gamma_{44}(q)\right]-\frac{1}{4} \gamma_{12}(q), \\
& S_{44}^{C}(q)=\frac{1}{8}\left[\gamma_{33}(q)+\gamma_{44}(q)\right]+\frac{1}{4} \gamma_{12}(q),
\end{aligned}
$$




$$
S_{12}^{C}(q)=\frac{1}{8}\left[\gamma_{11}(q)-\gamma_{22}(q)\right]
$$

Since the angular functions $\gamma_{i j}(q)$ can be computed numerically as described in detail by Amic et al., ${ }^{9}$ Eqs. (2)-(10) can be used to calculate the full Stokes reflection matrix for any $q$. If, furthermore, the transport mean free path $l$ is known, the reflection matrix can be expressed as a function of the phase angle $\alpha$ rather than the dimensionless angular parameter $q$. Note that the matrices $S^{L}(0)$ and $\mathbf{S}^{C}(0)$ given by Eqs. (4)-(10) fully comply with the general relationships derived for arbitrary macroscopically isotropic and symmetric scattering media and given by Eqs. (18) and (20)-(23) of Ref. 5.

Assuming unpolarized incident light $\left(Q_{0}=U_{0}=V_{0}=0\right)$, we define the intensity enhancement factor as the ratio of the total reflected intensity to the incoherent background value,

$$
\zeta(\alpha)=\frac{S_{11}^{1}(0)+S_{11}^{L}(0)+S_{11}^{C}(q)}{S_{11}^{1}(0)+S_{11}^{L}(0)} .
$$

Equations (3)-(5) show that only the first two Stokes parameters of the reflected light can be nonzero. Therefore, the degree of linear polarization can be defined as minus the ratio of the total reflected second Stokes parameter to the total reflected intensity:

$$
P(\alpha)=-\frac{Q(\alpha)}{I(\alpha)}=-\frac{S_{12}^{C}(q)}{S_{11}^{\perp}(0)+S_{11}^{L}(0)+S_{11}^{C}(q)} .
$$

Figure 1 shows both quantities as functions of the dimensionless angular parameter $q$. The upper panel demonstrates the renowned intensity peak centered at exactly the opposition. The amplitude of the peak is $\zeta(0) \approx 1.5368$ and its half-width at half-maximum is $q_{\zeta} \approx 0.597$. The former number agrees with the previous calculation reported in Table 2 of Ref. 5, whereas the latter number should replace the value 0.56 reported previously in Ref. 6 and derived using the asymptotic formulas of Ref. 8 . Thus the relationship between the half-width at half-maximum of the backscattering intensity peak and the photon transport mean free path for the case of conservative Rayleigh scattering and unpolarized normal illumination is given by

$$
\alpha_{5} \approx \frac{0.597}{k l}
$$


The lower panel of Fig. 1 shows that the reflected polarization is zero at opposition, which is a consequence of azimuthal symmetry in the case of normal illumination and unpolarized incident light. However, with increasing $q$, polarization becomes negative, rapidly grows in absolute value, and reaches its minimal value $P_{\min } \approx-2.765 \%$ at a reflection direction very close to opposition $\left(q_{P} \approx 1.68\right)$. The peak of negative polarization is highly asymmetric so that the half-minimal polarization value $-1.383 \%$ is first reached at $q_{P, 1} \approx 0.498$, which is even smaller than the value $q_{\zeta} \approx 0.597$ corresponding to the half-width at half-maximum of the backscattering intensity peak, and then at a much larger $q_{P, 2} \approx 7.10$. This unusual behavior of polarization at near backscattering angles was called in Ref. 6 the polarization opposition effect. To the best of our knowledge, the numbers $q_{\zeta} \approx 0.597, P_{\min } \approx-2.765 \%, q_{P} \approx 1.68, q_{P, 1} \approx 0.498$ and $q_{P, 2} \approx 7.10$ provide the first exact metrics of the photometric and polarization opposition effects caused by coherent backscattering of unpolarized incident light by a half-space of nonabsorbing Rayleigh scatterers.

\section{DISCUSSION}

Because lasers are the most frequently used sources of illumination and usually generate linearly or circularly polarized light, explicit laboratory demonstrations of the POE have been extremely rare. Apparently the first laboratory observation of the POE was made by Lyot as long ago as in the $1920 \mathrm{~s},{ }^{12}$ although the physical explanation of this phenomenon was, of course, unknown at that time. Figure 2 shows Lyot's polarization measurements for a particulate surface obtained by burning a tape of magnesium under a glass plate until the deposit on the plate was completely opaque. Lyot described the observed phase curve of polarization as "puzzling" and attributed it to the very small size of magnesia grains. Unfortunately, he did not measure the actual size of the grains and their packing density and, thus, did not provide the information necessary to compute the photon mean free path $l$. Furthermore, the minimal measured polarization value is only $-1.11 \%$, compared to the theoretical value $P_{\min } \approx-2.765 \%$ computed for Rayleigh particles. However, assuming that the latter difference is explained by the finite particle size in the Lyot's experiment and multiplying the theoretical polarization by a factor of 0.4 , and also assuming that the actual $k l$ was close to a realistic value of 132 . we were able to almost perfectly reproduce the angular profile of the mesaured polarization up to phase angles of about $15^{\circ}$ (solid curve in Fig. 
2). At larger phase angles the assumption of phase-angle-independent single-scattering and ladder contributions to the total reflection matrix [Eqs. (3) and (4)] is no longer valid and causes a significant deviation of the theoretical curve from the measurements.

The POE may have also been observed in more recent laboratory measurements by Geake and Geake ${ }^{13}$ for fine alumina powders, although the smallest phase angle in their experiments was $2^{\circ}$ and may be too large to make definitive conclusions. These measurements may suggest that the POE weakens and ultimately disappears with increasing particle size, which would be consistent with the similar disappearance of the spatial anisotropy of the backscattering intensity peak in the case of linearly polarized incident beam observed by van Albada et al. ${ }^{7}$

Sharp peaks of negative polarization at phase angles well less than a degree were observed for Saturn's rings and the Galilean satellites of Jupiter. ${ }^{5.14}$ Because these peaks were distinctly different from the symmetric, nearly parabolic negative polarization branch commonly observed for many solar system bodies, ${ }^{15}$ they were interpreted in terms of the POE produced by coherent backscattering of sunlight by a surface layer composed primarily of microscopic ice particles. ${ }^{5.14}$ The fact that the same objects exhibit comparably narrow intensity peaks centered at exactly the opposition ${ }^{16}$ provides a strong support for this interpretation.

As was emphasized previously, our present analysis is based on rigorous formulas of Ref. 9 and provides, for the first time, the exact metrics of the POE in the case of conservative Rayleigh scattering. Since the vector theory of coherent backscattering is extremely complicated and contains only a few rigorous results, ${ }^{5.9}$ we believe that our calculations can be useful in checking the accuracy of approximate approaches that are often based on multiple untested assumptions and have not been validated versus exact theories. ${ }^{17}$ Furthermore, it appears that laboratory and remote-sensing measurements of polarization at nearly zero phase angles can be an informative characterization and diagnostic tool in various physical and geophysical applications.

\section{ACKNOWLEDGMENT}

MIM acknowledges the support from the NASA Radiation Science Program managed by Dr. R. J. Curran. 


\section{REFERENCES}

1. Yu. N. Barabanenkov, Yu. A. Kravtsov, V. D. Ozrin, and A. I. Saichev, "Enhanced backscattering in optics," in Progress in Optics XXLX, E. Wolf, ed., (Elsevier, Amsterdam, 1991), pp. 65-197.

2. A. Lagendijk and B. A. van Tiggelen, "Resonant multiple scattering of light," Phys. Rep. 270, 143-215 (1996).

3. E. Amic, J. M. Luck, and Th. M. Nieuwenhuizen, "Anisotropic multiple scattering in diffusive media," J. Phys. A: Math. Gen. 29, 4915-4955 (1996).

4. M. C. W. van Rossum and Th. M. Nieuwenhuizen, "Multiple scattering of classical waves: microscopy, mesoscopy, and diffusion," Rev. Mod. Phys. 71, 313-371 (1999).

5. M. I. Mishchenko, "Enhanced backscattering of polarized light from discrete random media: calculations in exactly the backscattering direction," J. Opt. Soc. Am. A 9, 978-982 (1992).

6. M. I. Mishchenko, "On the nature of the polarization opposition effect exhibited by Saturn's rings," Astrophys. J. 411, 351-361 (1993).

7. M. P. van Albada, M. B. van der Mark, and A. Lagendijk, "Polarization effects in weak localization of light," J. Phys. D 21, S28-S31 (1988).

8. V. D. Ozrin, Exact solution for coherent backscattering of polarized light from a random medium of Rayleigh scatterers," Waves Rand. Media 2, 141-164 (1992).

9. E. Amic, J. M. Luck, and Th. M. Nieuwenhuizen, "Multiple Rayleigh scattering of electromagnetic waves," J. Phys. I France 7, 445-483 (1997).

10. M. I. Mishchenko, "Diffuse and coherent backscattering by discrete random media. 1. Radar reflectivity, polarization ratios, and enhancement factors for a half-space of polydisperse, nonabsorbing and absorbing spherical particles," J. Quant. Spectrosc. Radiat. Transfer 56, 673-702 (1996).

11. J. W. Hovenier and C. V. M. van der Mee, "Fundamental relationships relevant to the transfer of polarized light in a scattering atmosphere," Astron. Astrophys. 128, 1-16 (1983).

12. B. Lyot, "Research on the polarization of light from planets and from some terrestial substances," NASA Tech. Transl. F-187 (NASA. Washington, D. C., 1964).

13. J. E. Geake and M. Geake, "A remote-sensing method for sub-wavelength grains on planetary surfaces by optical polarimetry," Mon. Not. Roy. Astron. Soc. 245, 46-55 (1990). 
14. V. K. Rosenbush, V. V. Avramchuk, A. E. Rosenbush, and M. I. Mishchenko, "Polarization properties of the Galilean satellites of Jupiter: observations and preliminary analysis," Astrophys. J. 487, 402-414 (1997).

15. A. Dollfus, M. Wolff, J. E. Geake, D. F. Lupishko, and L. M. Dougherty, "Photopolarimetry of asteroids," in Asteroids II, R. P. Binzel, T. Gehrels, and M. S. Matthews, eds. (Univ. of Arizona Press, Tucson, AZ, 1989), pp. 594-616.

16. P. Helfenstein, N. Currier, B. E. Clark, J. Veverka, M. Bell, et al., "Galileo observations of Europa's opposition effect," Icarus 135, 41-63 (1998).

17. Yu. G. Shkuratov, "Interference model of the negative polarization of light scattered by the solid surfaces of celestial bodies," Astron. Vestnik 25, 152-161 (1991). 


\section{FIGURE CAPTIONS}

Fig. 1. Theoretical angular profiles of the intensity enhancement factor and the degree of linear polarization of the reflected light for a half-space of nonabsorbing Rayleigh particles illuminated by an unpolarized beam of light incident normally to the boundary of the scattering medium.

Fig. 2. Polarization measurements for a particulate surface composed of microscopic magnesia particles (dots) and best-fit theoretical computations (solid curve). 

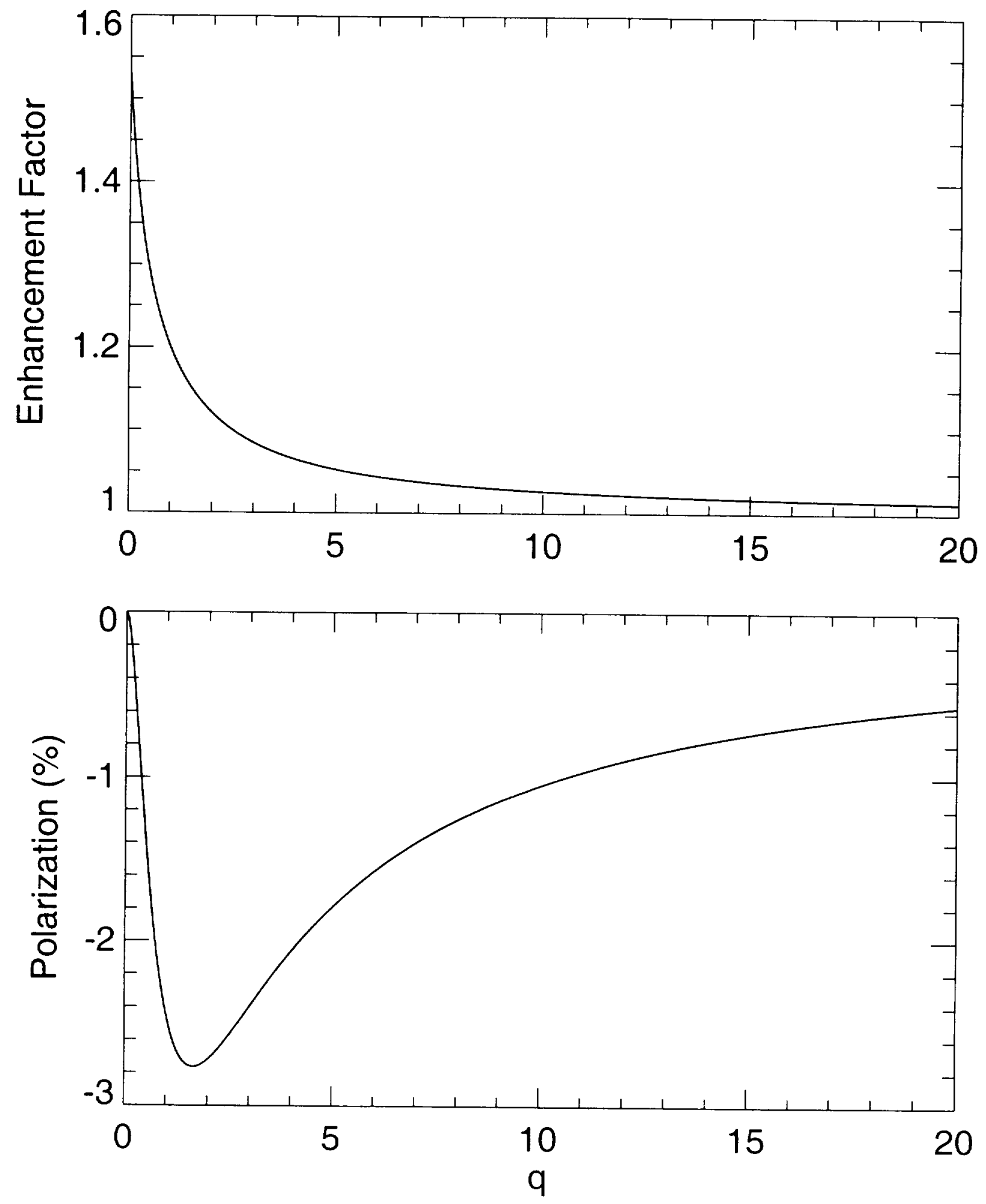

Fiq. I 


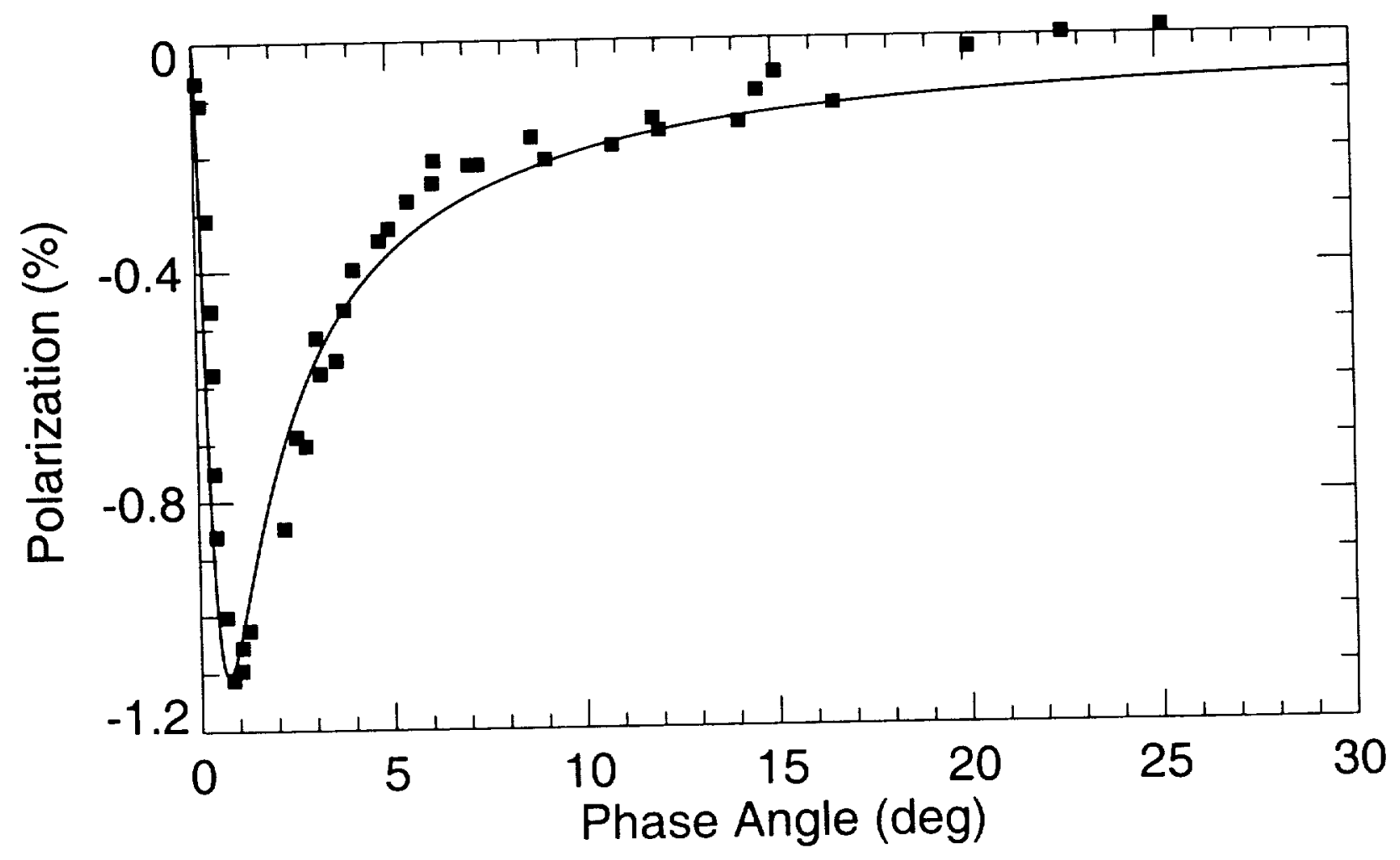

Fir. 2 